\title{
Cholesterol Monohydrate Dissolution Rate Studies in Aqueous Micellar Solutions of Polyoxyethylene Nonylphenol Ether and lonic Surfactants ${ }^{1}$
}

\author{
SHANKER L. GUPTA,* WILLIAM I. HIGUCHI, $\dagger^{2}$ AND NORMAN F. H. HO \\ ${ }^{*}$ E. R. Squibb, New Brunswick, New Jersey 08903; †Department of Pharmaceutics, College of Pharmacy, \\ University of Utah, Salt Lake City, Utah 84112; and \$College of Pharmacy, \\ The University of Michigan, Ann Arbor, Michigan 48109
}

Received April 17, 1983; accepted April 13, 1984

\begin{abstract}
The influences of electrical and other factors on the dissolution kinetics of cholesterol monohydrate in micellar solutions were quantified in terms of mass transfer resistances due to interfacial processes and convective/diffusional barriers. In Renex-690 (polyoxyethylene[10]nonylphenol ether) solutions, the mass transfer resistance, $R$, was large and constant over a wide concentration range of sodium chloride, indicating that neutral micelles were involved in slow interfacial kinetics via a collision complex mechanism. To study the effects of micellar charge and electrolyte, predetermined amounts of ionic surfactants were added to Renex-690 solutions. At constant Renex-690/benzalkonium chloride and Renex-690/sodium dodecyl sulfate ratios, curves of $R$ versus total counterion concentration were superimposable over a range of solubilizer concentration. Atso, $R$ decreased significantly with additions of $\mathrm{NaCl}$ and attained an asymptotic minimum which was expected for the convective/diffusion controlled case. These results were consistent with the collision complex mechanism and predictions based upon electrostatic interactions involving a charged sphere and a charged surface. The finding that there is a significant interfacial barrier with Renex-690 alone but not when both an ionic surfactant and sodium chloride were present was interesting. It is proposed that either the presence of the ionic surfactant in the mixed micelle may catalytically facilitate the transfer of the cholesterol molecule into the micelle or that the ionic surfactant adsorbed on the cholesterol crystal surface may be able to alter the accessibility of the cholesterol molecules on the crystal surface.
\end{abstract}

\section{INTRODUCTION}

The purpose of this study was to systematically examine the influence of electrical and other factors in the interfacially controlled dissolution kinetics of cholesterol monohydrate pellets in micellar solutions. The rationale has its origin in our numerous cholesterol monohydrate dissolution rate studies which were carried out in the presence of bile acid and bile acid/lecithin micellar solutions. These investigations have shown that the dissolution of cholesterol monohydrate crystals and human cholesterol gall-

\footnotetext{
${ }^{1}$ Work supported by NIAMDD Grants AM16694 and AM32472.

${ }^{2}$ To whom correspondence should be addressed.
}

stones may be limited by crystal/solution interfacial kinetics (1-4). Later, Kwan et al. (5) carried out a comprehensive study to establish the primary factors influencing the interfacially controlled dissolution in bile acid/lecithin solutions. Attempts have also been made to investigate the the questions as to how the interfacial barrier may be reduced by means of such additives as $n$ alkylamines and quaternary ammonium compounds (3-6). Subsequent studies supported the view that micellar charge neutralization by protonated amines was the primary way by which the alkylamines act as dissolution accelerators (7-9).

The problem of understanding the dissolution rate behavior of cholesterol monohydrate in bile acid/lecithin solutions is made 
complex by the fact that one has to deal with the coexistence of simple and mixed micelles (10-12). The ratios of the two micellar species may not be constant, for example, with changes in total concentration at a constant bile acid/lecithin ratio. Thus, it becomes difficult to describe the kinetics in terms of intrinsic "reactivity" of the particular micellar species involved. An additional complicating factor is that the behavior of bile acid micelles even in the absence of lecithin appears to be more complex in some ways compared to micellar behavior of conventional surfactants (13-16). For example, the micelle size (therefore, the average aggregation number) varies much more with concentration for bile acid solutions than for conventional surfactants.

In the following studies, Renex- 690 (polyoxyethylene[10]nonylphenol ether) was selected as the nonionic surfactant. Here, one is dealing with a typical, high-purity surfactant with a sharp CMC and a monomer concentration that is essentially constant over a concentration range $(17,18)$. Thus, it was anticipated that the "collision complex" hypothesis could be tested in a more systematic way. Furthermore, it was anticipated that with the nonionic micellar system the influence of the extent of micellar charge on the interfacial kinetics could be investigated by the addition of predetermined amounts of ionic surface active agents to Renex-690 solutions. Also, early studies showed that nonionic Renex-690 micelles themselves may be associated with slow interfacial kinetics; therefore, this offered the opportunity for investigating factors other than electrical charge that may influence the interfacial barrier.

\section{EXPERIMENTAL}

\section{Static Disk Dissolution Method}

The dissolution apparatus is schematically illustrated in Fig. 1. The design of the system provides a high-dissolution surface area-tovolume ratio to significantly shorten the ex-

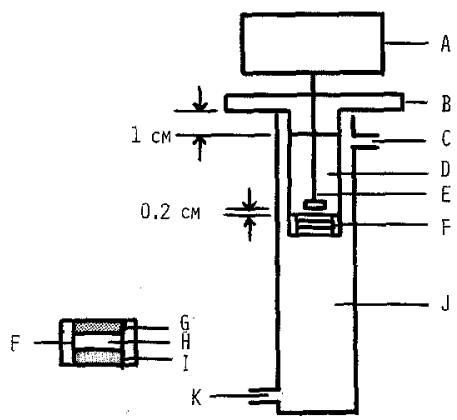

FIG. 1. Diagram of the static pellet dissolution apparatus. Key: $A$, synchronous motor $(150 \mathrm{rpm}): \mathrm{B}$, Delrin cover-mount; $C$, water outlet; $D$, dissolution chamber (15 ml); E, Delrin stirrer; F, Delrin die-pellet holder; G, cholesterol pellet; H, punch; I, paraffin wax; J, outer water jacket; $\mathbf{K}$, water inlet.

perimental period and reduce the cost of expensive reagents.

The reagents included cholesterol monohydrate,${ }^{3}\left[{ }^{14} \mathrm{C}\right]$ cholesterol monohydrate, ${ }^{4} \mathrm{Re}-$ nex- $690,{ }^{5}$ sodium dodecyl sulfate, ${ }^{3}$ benzalkonium chloride, ${ }^{3}$ (benzyldimethyltetradecylammonium chloride), and sodium chloride. Cholesterol was recrystallized three times with 95\% ethanol. Radioactive cholesterol monohydrate crystals were prepared by mixing 5 $\mathrm{g}$ of the recrystallized cholesterol with 100 $\mu \mathrm{Ci}$ of a benzene solution of $\left[{ }^{14} \mathrm{C}\right]$ cholesterol in $400 \mathrm{ml}$ of $95 \%$ ethanol at $60^{\circ} \mathrm{C}$. The solution was filtered while hot and the filtrate was allowed to stand for $48 \mathrm{hr}$ at room temperature. Only crystals obtained from slow crystallization after $12 \mathrm{hr}$ were used. The crystals were filtered and dried in vacuo for $24 \mathrm{hr}$. They were stored in the dark in a dessicator saturated with water vapor at room temperature, checked for purity by TLC (19) and discarded if any degradation products were noticed. This procedure, as developed in our laboratory, has been shown to yield cholesterol monohydrate crystals with a lattice system similar to that of cholesterol found in human biliary calculi $(5,6,20)$.

Renex-690, a polyoxyethylene[10]nonyl-

\footnotetext{
${ }^{3}$ J. T. Baker, Chemical Co., Philipsburg, N. J.

${ }^{4}$ New England Nuclear Corp., Boston, Mass.

${ }^{5}$ ICI Americas Inc., Wilmington, Del.
} 
henol ether, was used as received. The average ethylene oxide chain length was 10 with a chain length distribution mainly in the range 8 to 12 . The material was believed to contain negligible amounts of unreacted nonylphenol and only minor amounts $(\sim 1-2 \%)$ of free polyethylene glycols. ${ }^{6}$ An analysis ${ }^{7}$ showed no detectable sodium, halogens, or a residue after ashing. Benzalkonium chloride, sodium dodecyl sulfate, and sodium chloride were of analytical grade and were used as received.

Pellets of ${ }^{14} \mathrm{C}$-labeled cholesterol monohydrate crystals were prepared by compressing $50-100 \mathrm{mg}$ of the material in the Delrin die, 0.5 in. i.d., under $455 \mathrm{~kg}$ pressure using a laboratory press. $^{8}$ The exposed surface area of the pellet was $1.267 \mathrm{~cm}^{2}$. Exactly $2 \mathrm{ml}$ of the dissolution medium, containing Renex690 in the absence or presence of varying concentrations of ionic surfactants in deionized water ${ }^{9}$ and preequilibrated at $37^{\circ} \mathrm{C}$, was added. Stirring was achieved with a 150 -rpm synchronous motor ${ }^{10}$ with the Delrin stirrer placed $0.2 \mathrm{~cm}$ above the tablet surface. Microliter samples were withdrawn at suitable time intervals and assayed using a liquid scintillation counter. ${ }^{11}$

\section{Diffusion Coefficient and Binding Determinations}

Diffusion coefficients of micelles containing $\left[{ }^{14} \mathrm{C}\right]$ cholesterol were determined at $37^{\circ} \mathrm{C}$ in a small volume, two-compartment, Lucite diffusion cell by the method similar to that described by Krevoy and Wewerka (21) and Keller et al. (22). A silver filter membrane $(0.45-\mu \mathrm{m} \text {-diameter pore size })^{12}$ separated the two well-stirred compartments. The mem-

\footnotetext{
${ }^{6}$ G. J. Stockburger, ICI Americas Inc., Wilmington, Del. (personal communication).

${ }^{7}$ Performed by M-H-W Laboratories, Phoenix, Ariz.

${ }^{8}$ Model B, Fred Carver, Inc., Summit, N. J.

${ }^{9}$ Millipore Corp., Bedford, Mass.

${ }^{10}$ Model CA, Hurst Mfg. Co., Princeton, N. J.

" Model LS 9000, Beckman Instruments Inc., Fullerton, Calif.

${ }^{12}$ Selas Flotronics, Huntington Valley, Pa.
}

brane was boiled before each experiment in deionized water for $30 \mathrm{~min}$ in order to remove entrapped air. When formation of black silver oxide occurred, a new silver membrane was used since the transport rate was affected. The cell constant was determined by the use of $\left[{ }^{14} \mathrm{C}\right]$ sucrose $^{4}$ in water whose diffusion coefficient $\left(7.05 \times 10^{-6} \mathrm{~cm}^{2} / \mathrm{sec}\right.$ at $37^{\circ} \mathrm{C}$ at infinite dilution) is well known (23-25).

To estimate the degree of binding of ionic surfactant to Renex-690 micellar solutions, a dynamic membrane dialysis technique (26) was utilized. The surfactant system was contained in a dialysis membrane bag. ${ }^{13}$ The free benzalkonium chloride appearing outside the bag in a $37^{\circ} \mathrm{C}$ thermostated beaker was sampled with time and assayed spectrophotometrically at $208 \mathrm{~nm}$. The permeation constant of the membrane system was determined with sodium benzoate by spectrophotometric assay at $225 \mathrm{~nm}$. There were no adverse effects on the membrane property by the surfactants throughout the course of the experiments.

\section{Data Treatment}

From the plot of the concentration of cholesterol dissolved in the bulk micellar solution versus time, the data was analyzed using the following rate equation to account for both the interfacial resistance and the aqueous diffusional resistance in the dissolution process:

$$
\frac{J}{A}=\frac{V}{A}\left(\frac{d C_{\mathrm{b}}}{d t}\right)=\frac{C_{\mathrm{s}}-C_{\mathrm{b}}}{R}
$$

where $J$ is the dissolution rate; $V$ is the volume of dissolution media; $A$ is the surface area of dissolving solid; $C_{\mathrm{s}}$ is the concentration of cholesterol in solution at saturation; $C_{\mathrm{b}}$ is the concentration in the bulk medium (under sink conditions, $\left.C_{\mathrm{b}}=0\right) ; R$ is the total resistance to dissolution.

\footnotetext{
${ }^{13}$ Spectrapor, MW cutoff 6000-8000, Spectrum Medical Industries, Inc., Los Angeles, Calif.
} 


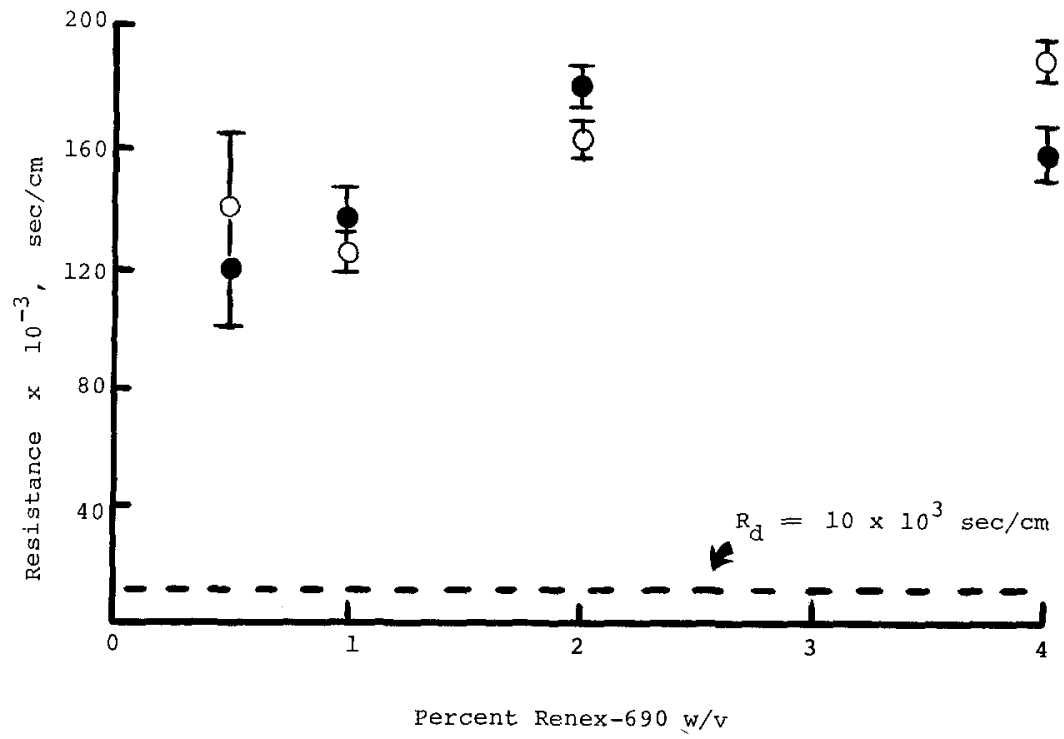

FIG. 2. Total transport resistance as a function of Renex -690 concentration in the presence and absence of $0.1 \mathrm{M}$ sodium chloride. Key: $\mathrm{O}$, control; $\bullet, 0.1 \mathrm{M}$ sodium chloride. The dashed line represents the mass transfer resistance for the limiting convective/diffusion-controlled mechanism.

The total resistance is the sum of two resistances (27):

$$
R=\frac{h}{D}+\frac{1}{P}
$$

where $h$ is the effective aqueous diffusion layer thickness, $D$ is the aqueous diffusion coefficient of the micelle, and $P$ is the effective permeability coefficient of the interfacial barrier. In many cases, $1 / P$ has been found to be much greater than $h / D$; that is, the dissolution is surface-controlled. When $1 / P$ is much smaller than $h / D$, the dissolution is diffusion-controlled.

The integration of Eq. [1] over discrete time intervals gives

$$
\ln \frac{C_{\mathrm{s}}-C_{\mathrm{b} 1}}{C_{\mathrm{s}}-C_{\mathrm{b} 2}}=\frac{A\left(t_{2}-t_{1}\right)}{V_{\mathrm{t} 1} R}
$$

where $C_{\mathrm{b} 1}$ is the concentration in the bulk at time $t_{1} ; C_{\mathrm{b} 2}$ is the concentration in the bulk at time $t_{2}$; and $V_{\mathrm{t} 1}$ is the volume of the dissolution media at the time the sample for $C_{\mathrm{b} 2}$ determination was taken. Rearranging Eq. [3] we get

$$
\begin{aligned}
C_{\mathrm{b}, 2}=C_{\mathrm{s}}+\left(C_{\mathrm{b} 1}\right. & \left.-C_{\mathrm{s}}\right) \\
& \times \exp \left[-\frac{A\left(t_{2}-t_{1}\right)}{V_{\mathrm{t} 1} R}\right] .
\end{aligned}
$$

Equation [4] was utilized to determine $R$ and $C_{\mathrm{s}}$ simultaneously by interactive nonlinear least-square curve fitting on a digital computer. ${ }^{14}$

\section{RESULTS}

\section{Dissolution Studies in Renex-690}

Figures 2 and 3 indicate that sodium chloride has no effect on both $R$ and $C_{\mathrm{s}}$ on the dissolution kinetics in 1\% Renex-690. Moreover, the $R$-values are relatively independent of Renex-690 concentration, even with an eight-fold change in concentration. The solubility of cholesterol monohydrate is proportional to the amount of solubilizer present. It is seen in Fig. 2 that the total mass transfer resistance is about 12 to 18 times greater

${ }^{14}$ Dr. J. L. Fox, Department of Pharmaceutics, University of Utah. 


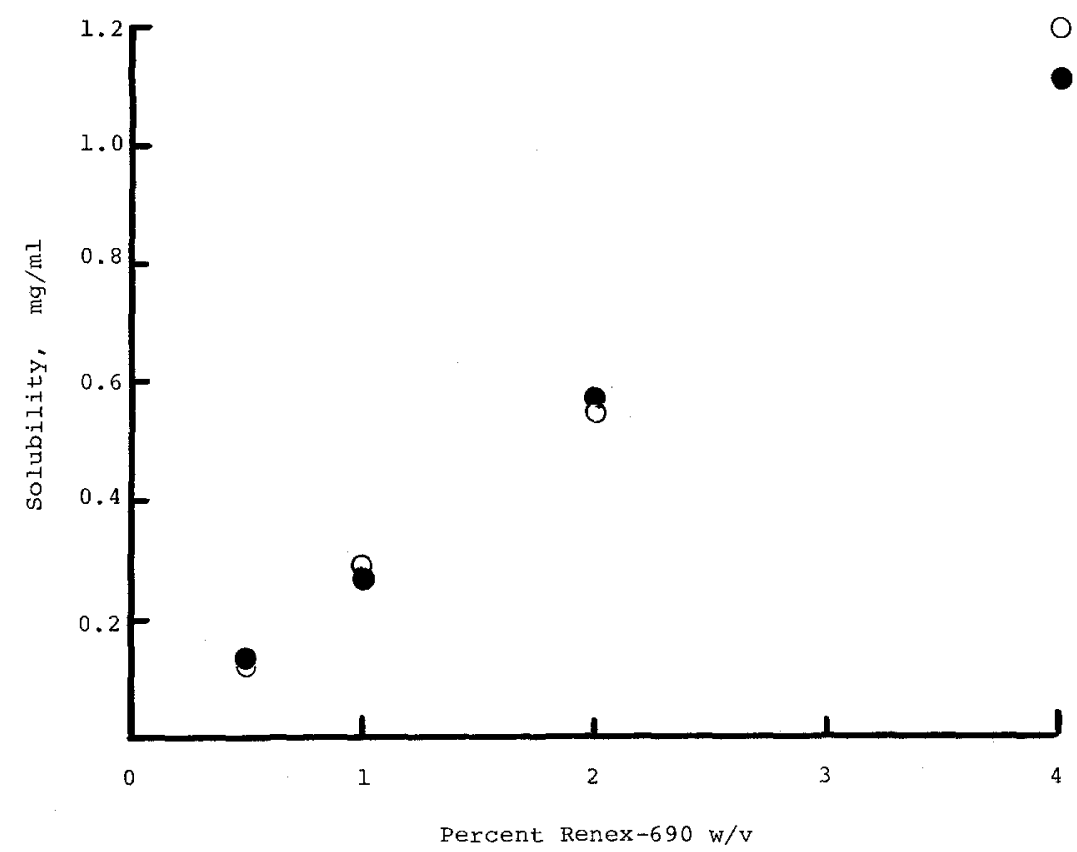

FIG. 3. Influence of Renex-690 concentration on cholesterol monohydrate solubility. Key: $O$, control; , $0.1 M$ sodium chloride.

than that expected for the diffusion-controlled resistance as indicated by $R_{\mathrm{d}}$, which is equal to $h / D$. The $R_{\mathrm{d}}$ value of $10 \times 10^{3} \mathrm{sec} / \mathrm{cm}$ was established from subsequent studies involving micellar systems of Renex-690 and ionic surfactants.

\section{Dissolution Studies on Renex-690 Solutions Containing Benzalkonium Chloride (BC)}

Figures 4-8 present data at varying concentrations of Renex-690 and BC, showing the influences of the added $\mathrm{BC}$ and sodium chloride at $1 \%$ Renex-690 and a constant Renex/BC weight ratio of 4.0 upon $R$, the transport resistance, and $C_{\mathrm{s}}$, the solubility. The influences of both $\mathrm{BC}$ and sodium chloride upon $C_{\mathrm{s}}$ are seen to be modest (Fig. 5), being at most a factor of two over the concentration range studied. In contrast, the influences of both of these additives upon $R$ were very large (Fig. 4). At low or zero sodium chloride, $R$ increases by about a factor of 10 over the concentration range of
$\mathrm{BC}$ investigated. At the other extreme, i.e., when the sodium chloride concentration was $0.1 M, \mathrm{R}$ decreases steadily with increasing BC concentration until at $6.8 \mathrm{mM}$ (corresponding to $0.25 \% \mathrm{w} / \mathrm{v}) \mathrm{BC}$, the $R$ value is about 10 times smaller than that for no $\mathrm{BC}$ present.

Figures 6 and 8 show the simultaneous influences of the total solubilizing agent (Renex-690 and BC) and sodium chloride at the constant Renex-690-to-BC ratio of 4.0. It is seen that $C_{\mathrm{s}}$ is only weakly influenced by the electrolyte for all levels of the solubilizing agent. Also, at all sodium chloride concentrations, the $C_{\mathrm{s}}$ values are seen to be approximately proportional to the amount of the solubilizing agent present, suggesting that the size, shape, and/or structure of the micelles do not greatly change over the concentration range. The $R$ values show a strong dependence upon electrolyte covering about a 50to 100 -fold range (Fig. 6). $R$ is most sensitive to sodium chloride concentrations ranging 


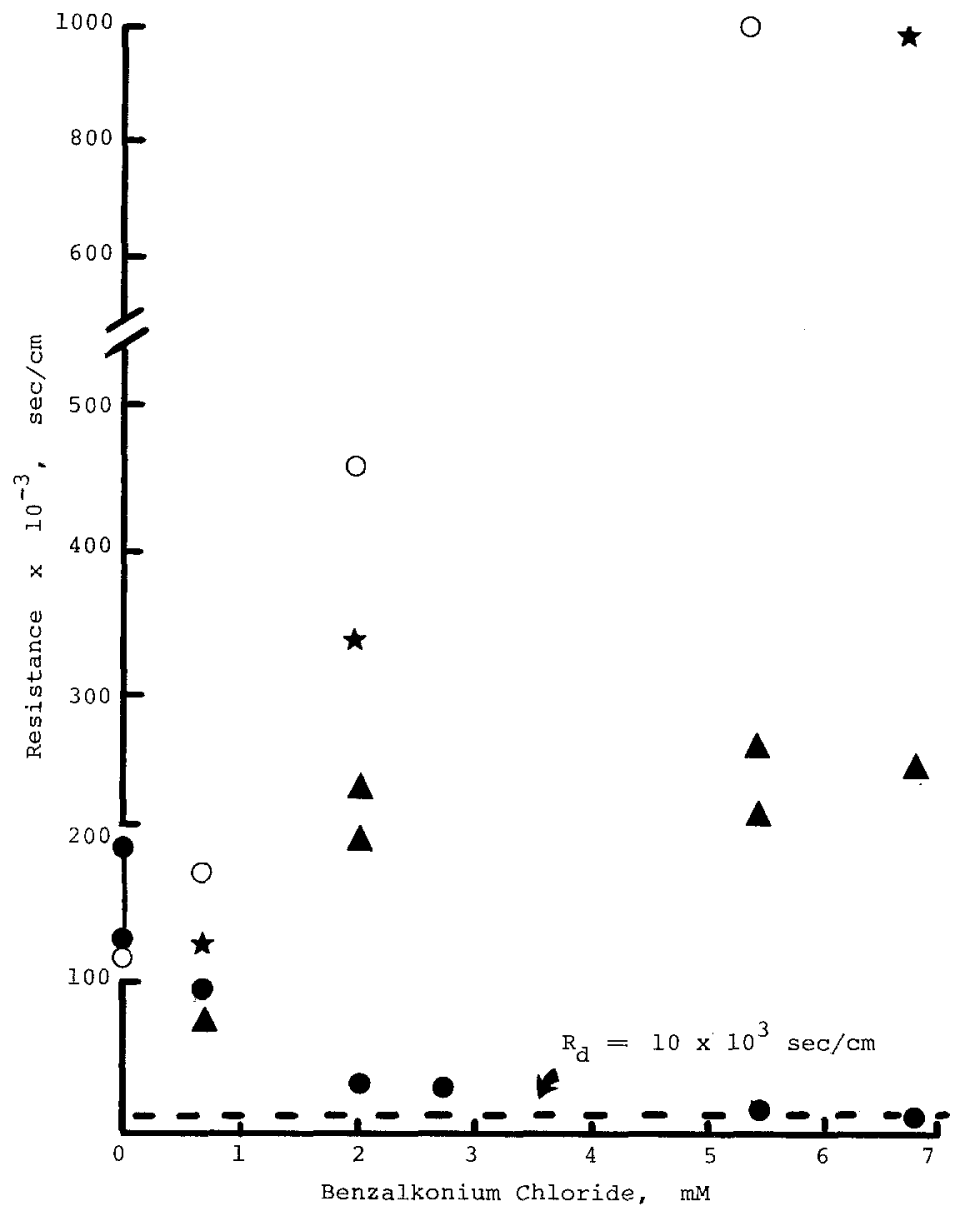

FIG. 4. Influence of benzalkonium chloride in 1\% Renex-690 on the total transport resistance at several concentrations of sodium chloride. Key: $O$, control; $\star, 0.01 M$ sodium chloride; $\wedge, 0.03 M$ sodium chloride; $0.1 \mathrm{M}$ sodium chloride.

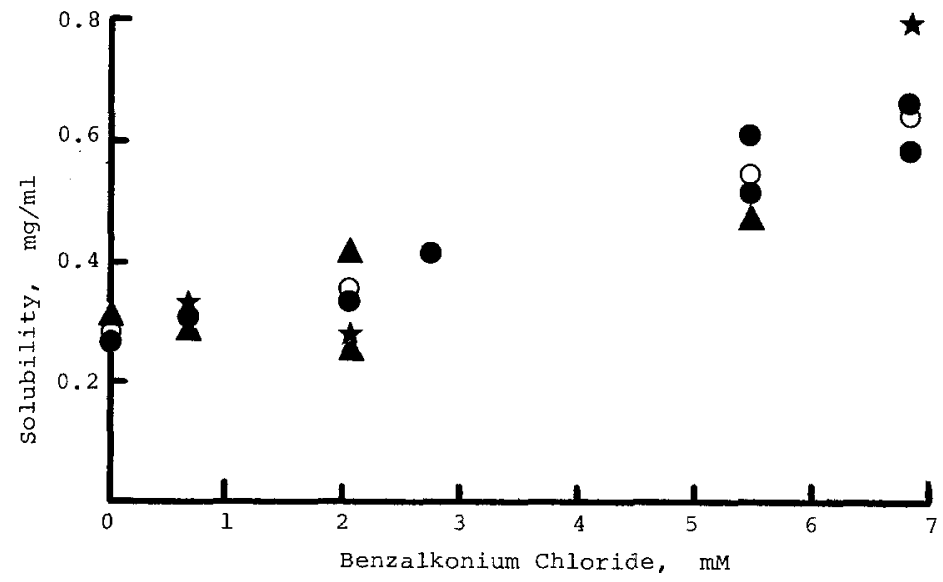

FIG. 5. Cholesterol monohydrate solubility in 1\% Renex-690 solutions containing benzalkonium chloride and sodium chloride. Key: $\mathrm{O}$, control; $\star, 0.01 M$ sodium chloride; $\wedge, 0.03 \mathrm{M}$ sodium chloride; , $0.1 M$ sodium chloride. 


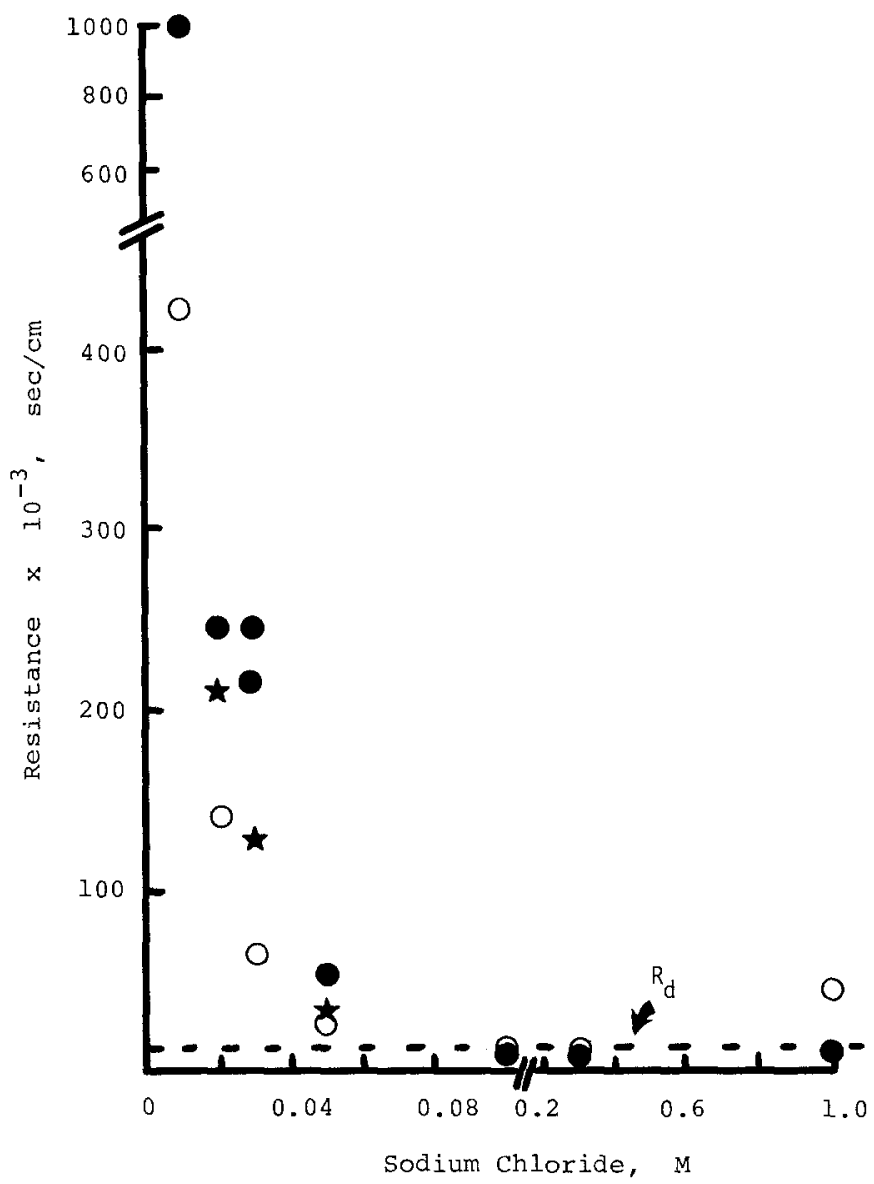

FIG. 6. Influence of sodium chloride concentration on transport resistance at several levels of total solubilizer concentration. Renex-690-to-benzalkonium chloride weight ratio was kept constant at 4.0. Key: •, 1\% Renex-690, 6.8 mM BC; $\star, 2 \%$ Renex-690, $13.6 \mathrm{~m} M \mathrm{BC} ;$ O, 4\% Renex-690, $27.2 \mathrm{~m} M \mathrm{BC}$.

from 0.01 to $0.1 M$ and becomes relatively constant in the range of 0.1 to $1.0 \mathrm{M}$. For the $4 \%$ Renex-690 case, however, there is a sudden increase in $\mathrm{R}$ at $1.0 \mathrm{M}$ sodium chloride.

Figure 7 is the replot of the data in Fig. 6 in terms of total chloride concentration contributed by $\mathrm{BC}$ and $\mathrm{NaCl}$. It is seen that the data generally are superimposable up to 0.3 $M$ total chloride. Thus, the total counterions rather than simply the added electrolyte determine the kinetics. Dialysis experiments indicate that more than $98 \%$ of $\mathrm{BC}$ is bound with the Renex-690 micelles resulting in the formation of positively charged micelles of mixed composition.
It is clear that $R$ reaches a minimum value $\left(10 \times 10^{3} \mathrm{sec} / \mathrm{cm}\right)$ which is independent of counterion concentration and indicative of a diffusion-controlled situation. In Table I the diffusion coefficients of various micellar systems are nearly identical, ranging between $4.7 \times 10^{-7}$ and $6 \times 10^{-7} \mathrm{~cm}^{2} / \mathrm{sec}$. Using $R$ $=h / D$, it is found that the effective aqueous boundary layer thickness, $h$, is about $50 \mu \mathrm{m}$ which is very reasonable for the well-stirred condition employed here. Consequently, the limiting resistance is designated as $R_{\mathrm{d}}$, the mass transfer resistance for the diffusioncontrolled case, and is useful in delineating the resistance of the interfacial barrier from the total resistance. 


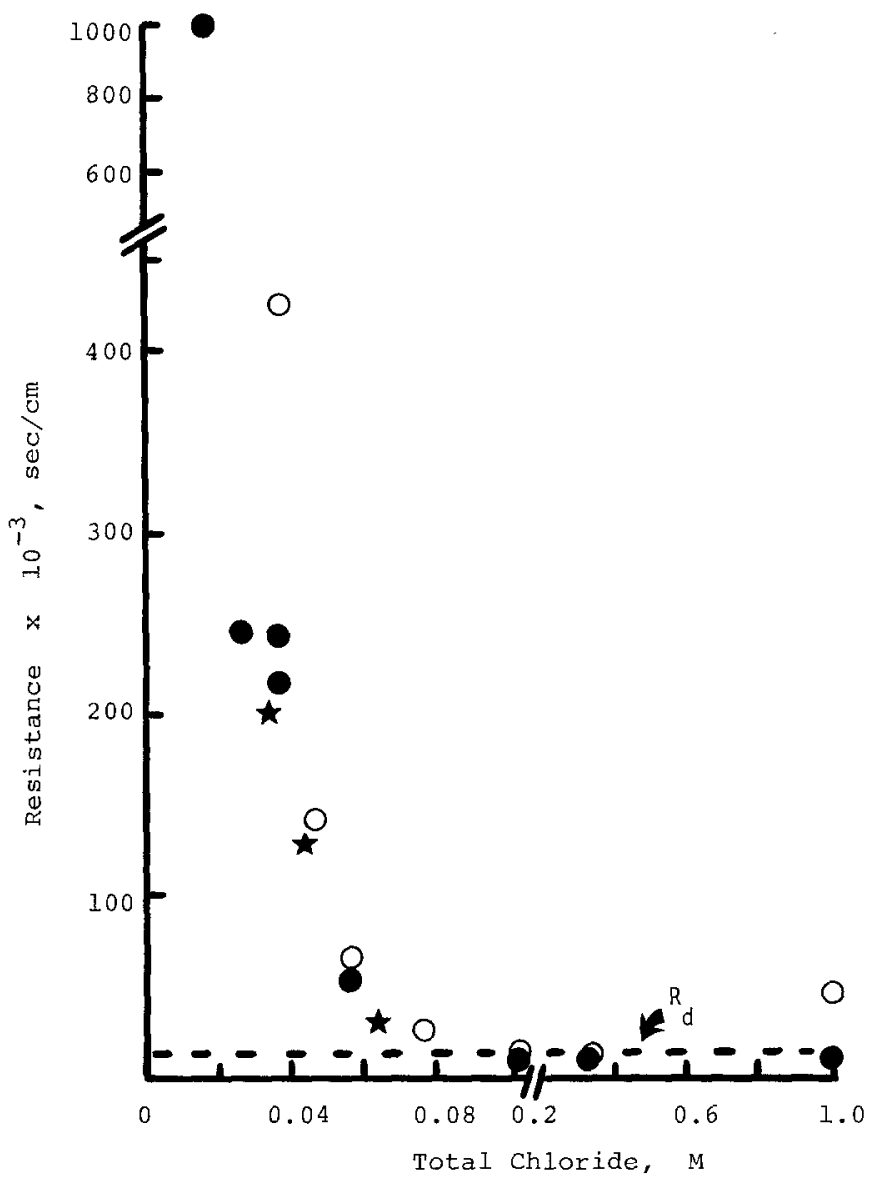

FIG. 7. Replotting of data in Fig. 6 with total counterion (chloride) concentration for the abscissa. Key: •, $1 \%$ Renex-690, 6.8 mM BC; $\star, 2 \%$ Renex-690, $13.6 \mathrm{~m} M \mathrm{BC} ; \mathrm{O}, 4 \%$ Renex-690, $27.2 \mathrm{~m} M \mathrm{BC}$.

Dissolution Studies in Renex-690 Solutions Containing Sodium Dodecyl Sulfate (SDS)

Figure 9 shows the results of experiments at varying concentrations of Renex-690 and SDS at the constant Renex-690-to-SDS weight ratio of 5.0. As reported earlier for the Renex$690 / \mathrm{BC}$ system, the $R$ values are strongly dependent upon the sodium chloride concentration varying up to 100 -fold in range. $R$ follows a single "function" when total $\mathrm{Na}$ counterion concentration is plotted for the abscissa. The $C_{\mathrm{s}}$ values in Fig. 10 are seen to be proportional to the amount of total solubilizer present. These data, together with the similar results for the Renex-690/BC system, suggest that the total counterion concentra- tion rather than simply the added electrolyte must be taken into account. It is noted that the limiting resistance is $10 \times 10^{3} \mathrm{sec} / \mathrm{cm}$, the same as that found for the BC system.

Although binding experiments were not carried out with SDS in the presence of Renex-690, it is reasonable on the basis of the binding studies with $\mathrm{BC}$ to assume that SDS is also more than $98 \%$ bound with Renex-690 micelles.

\section{DISCUSSION}

\section{Collision Complex versus Other Mechanisms}

The experimental results strongly support the hypothesis that the rate-determining step 


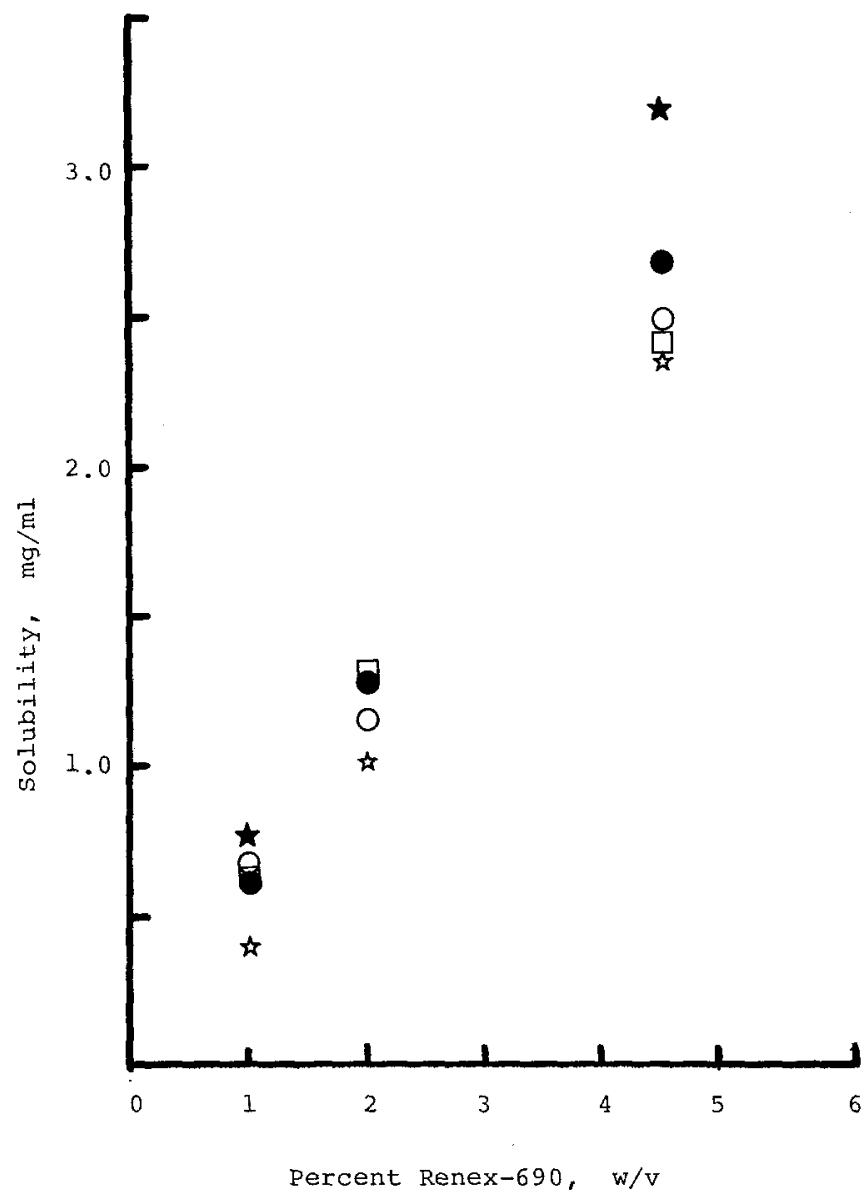

FIG. 8. Cholesterol monohydrate solubility as a function of total solubilizer concentration for several sodium chloride concentrations. Renex-690-to-benzalkonium chloride weight ratio kept constant at 4.0. Key: $*, 0.02 M \mathrm{NaCl} ; \square, 0.03 \mathrm{M} \mathrm{NaCl} ; 0,0.05 \mathrm{M} \mathrm{NaCl} ; \bullet, 0.10 \mathrm{M} \mathrm{NaCl}$;, $1.0 \mathrm{M} \mathrm{NaCl}$.

in cholesterol monohydrate dissolution in the nonionic surfactant system involves the transport of micellar cholesterol at the interface rather than a free solute mechanism.

In the case of Renex-690 alone, the interfacial resistance $R$ is found to be relatively constant over a wide concentration range with and without sodium chloride (Fig. 2). This constancy of $R$ supports the micelle collision mechanism since the free solute mechanism would be expected to show a direct proportionality between $R$ and solubilizer concentration. The solubility of cholesterol in these solutions is approximately proportional to the total amount of solubilizer present (Fig. 3) indicating that the basic nature of the Renex micelle does not vary greatly over the concentration range studied.

In the cholesterol monohydrate dissolution rate studies at constant Renex-690/BC and Renex-690/SDS surfactant ratios, when the counterion contribution from the ionic surfactant is taken into account, the $R$ versus total counterion curves were found to be essentially superimposable over a range of the solubilizer concentration (Figs. 7 and 9). These experimental results are again consistent with the micelle collision mechanisms 
TABLE I

Diffusion Coefficients of Various Micelles Containing Cholesterol in Water at $37^{\circ} \mathrm{C}$

\begin{tabular}{lc}
\hline \multicolumn{1}{c}{ Solution } & $\begin{array}{c}D \times 10^{7}, \mathrm{~cm}^{2} / \mathrm{sec} \\
( \pm \mathrm{SD})\end{array}$ \\
\hline $\begin{array}{l}\text { 1\% Renex-690 } \\
\text { 4\% Renex-690 } \\
1 \% \text { Renex-690,6.8 mM }\end{array}$ & $5.55 \pm 0.45$ \\
$\quad$ SDS, $0.3 M \mathrm{NaCl}$ & $6.17 \pm 0.42$ \\
1\% Renex-690,6.8 mM BC, & $4.68 \pm 0.24$ \\
$0.3 M \mathrm{NaCl}$ & \\
\hline
\end{tabular}

and predictions from colloid theory for the interaction between a charged sphere and a charged cholesterol surface.
Nature of the Interfacial Barrier

Electrical property. The dissolution phenomena of cholesterol monohydrate pellets in the presence of Renex-690/ionic surfactant systems indicate that, in part, the interfacial barrier is electrical in nature. The dissolution process involves collisional kinetics of the charged, cholesterol-free micelles with the charged cholesterol surface after overcoming the electrostatic potential energy barrier in accordance with the classical DLVO (Derjaguin, Landau, Verwey, and Overbeek) theory for interacting charged surfaces. The addition of counterions reduces the electrical repulsion and, consequently, the mass transfer resistance $R$ where, in the limit, the kinetics becomes diffusion/convection-controlled.

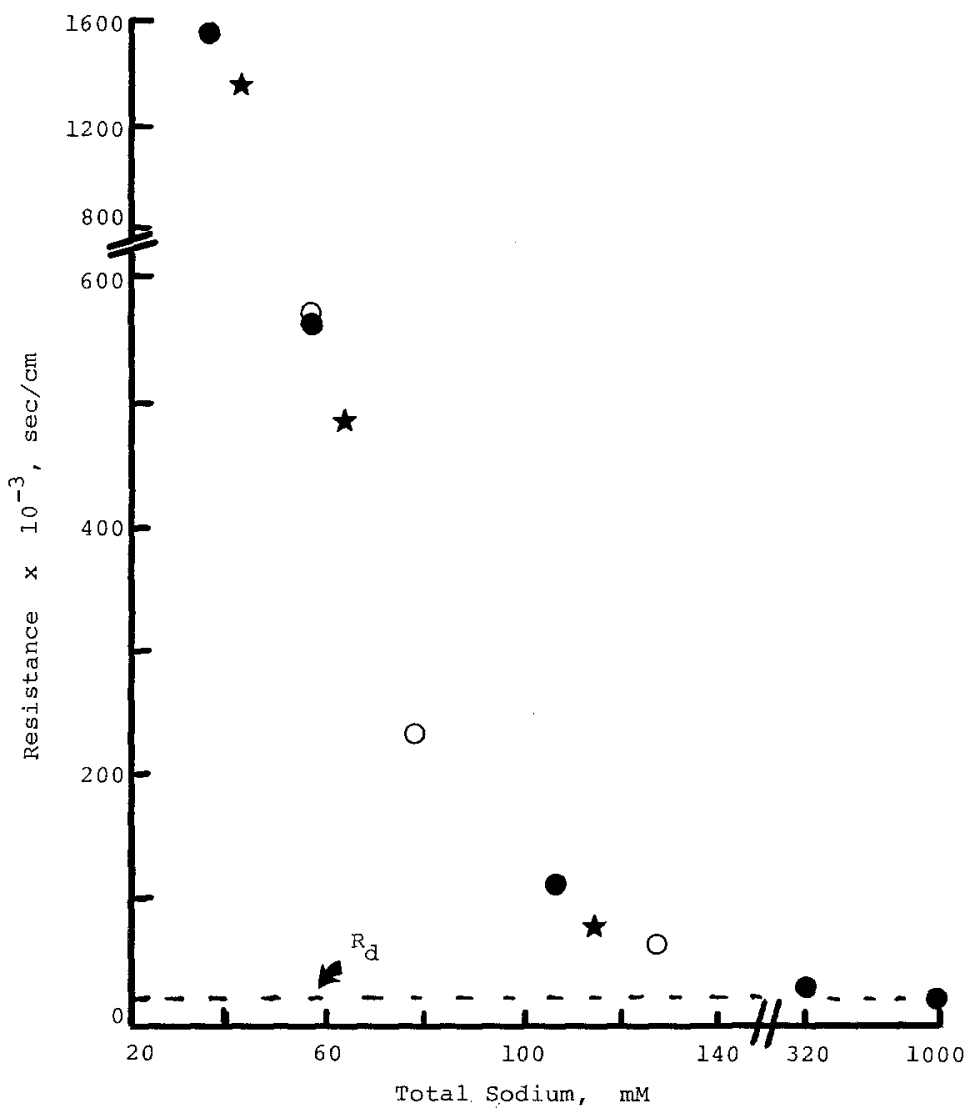

FIG. 9. Influence of total sodium counterion concentration on cholesterol dissolution kinetics. Key: $\bullet$, 1\% Renex-690, 6.8 m $M$ SDS; $\star, 2 \%$ Renex-690, 13.6 $\mathrm{m} M$ SDS; $O$, 4\% Renex-690, $27.2 \cdot \mathrm{m} M$ SDS. 


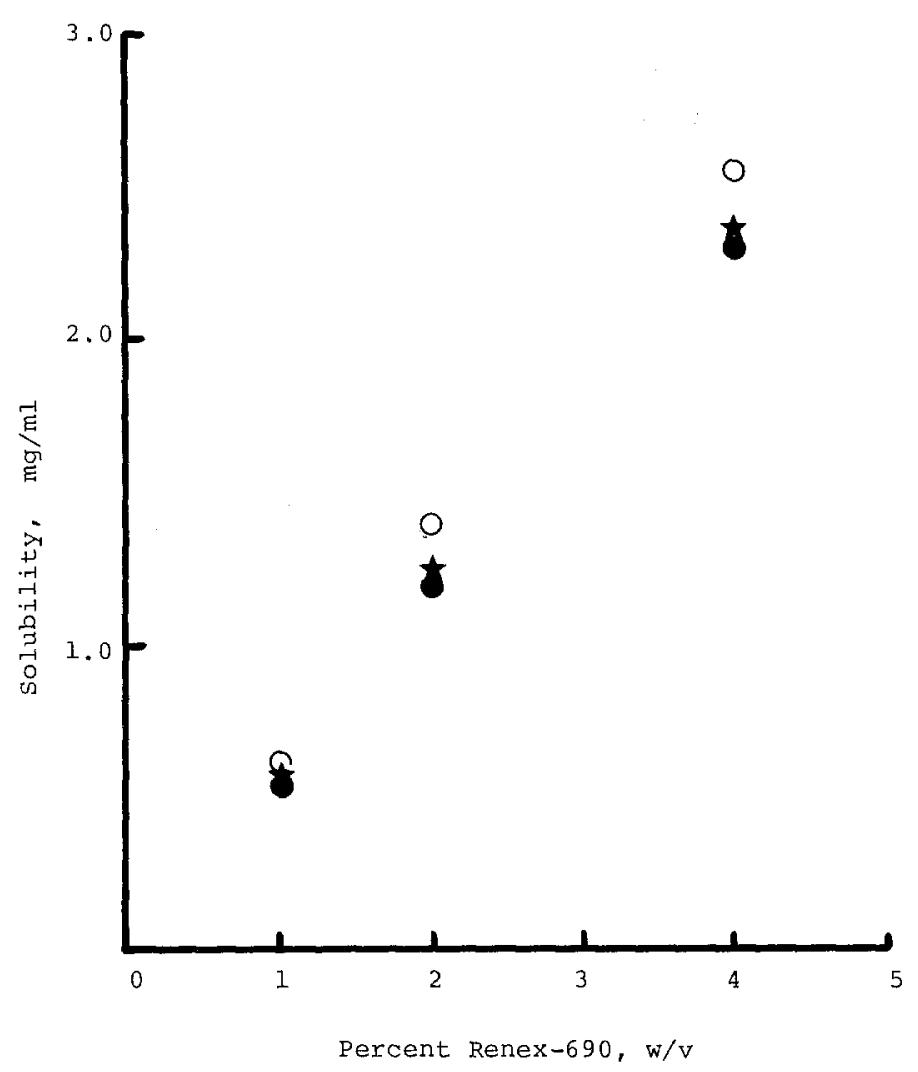

FIG. 10. Cholesterol monohydrate solubility as a function of total solubilizer concentration for several sodium chloride concentrations. Renex-690-to-sodium dodecyl sulfate ratio kept constant at 5.0. Key: $\bullet$ $0.05 \mathrm{M} \mathrm{NaCl} ; \star, 0.1 \mathrm{M} \mathrm{NaCl} ; 0,0.3 \mathrm{M} \mathrm{NaCl}$.

Nonelectrical factors. There is strong evidence that electrostatic factors at the interface are not the only ones involved in the interfacially controlled dissolution of cholesterol in aqueous micellar solutions. First, Renex690 alone (a neutral micelle) has a substantial interfacial barrier. The total transport resistance, $R$, is about 15 -fold greater than that predicted for aqueous boundary layer-controlled dissolution. The lack of influence of $\mathrm{NaCl}$ shows that the $R$ value for this situation is not due to ionic impurities giving rise to electrostatic repulsion. Second, the results show that ionic surface-active agents are able to significantly reduce the $R$ values. The questions, therefore, arise as to why the neutral Renex-690 micelle is associated with a substantial interfacial barrier and why added charged surfactants plus electrolyte are able to enhance the kinetics of cholesterol dissolution beyond the maximum rates found with Renex-690 alone. These questions may be examined by considering factors associated with the micelle and other possible factors associated with cholesterol crystal surface.

Nonionic Renex-690, containing polyoxyethylene chains and a 9-carbon head group, form micelles in aqueous solutions which consist of a hydrophobic core surrounded by a voluminous mantle of highly hydrated polyoxyethylene chains (28-31). It would then be expected that solubilizates, depending on their structure and polarity, would be preferentially distributed either in the hydrophobic core or in the outer polyoxyethylene mantle $(29,32,33)$. Also, one might expect that hydrophobic molecules such as cholesterol will be predominantly (on a time 
average basis) distributed in the hydrophobic region and that the transfer kinetics of cholesterol from a crystal surface to the micelle might be limited by movement through the hydrated polyoxyethylene mantle. If this were true, additives which would preferentially partition into the mantle and interact with cholesterol during transit may be able to catalyze the dissolution rate. This viewpoint is consistent with the dissolution rate enhancement found with $\mathrm{BC}$ and SDS. One may speculate that a charged surfactant together with its counterion is more likely to be preponderant in the hydration mantle.

The above mechanism might be considered an attractive one for explaining the large amount of data in this research. Other evidence suggests, however, that this may not necessarily be the entire interpretation. Feld et al. (34) have shown that the interfacial kinetics of cholesterol monohydrate in bile acid solutions was dependent not only upon micelle characteristics but also upon the nature of the solid surface. Here, the interfacial events were explained by a concerted twostep reaction involving the disengagement kinetics of cholesterol monohydrate molecules and the collision of the micelle with the surface. Thus, it is conceivable the amphipathic surfactants used in this present study could alter the surface properties of the cholesterol monohydrate crystals in some way.

\section{REFERENCES}

1. Higuchi, W. I., Prakongpan, S., Surpuriya, V., and Young, F., Science 178, 633 (1972).

2. Higuchi, W. I., Sjuib, E., Mufson, D., Simonelli, A. P., and Hofmann, A. F., J. Pharm. Sci. 62, 942 (1973).

3. Higuchi, W. I., Prakongpan, S., and Young, F., $J$. Pharm. Sci. 62, 1207 (1973).

4. Prakongpan, S., Higuchi, W. I., Kwan, K. H., and Molokhia, A. M., J. Pharm. Sci. 65, 685 (1976).

5. Kwan, K. H., Higuchi, W. I., Molokhia, A. M., and Hofmann, A. F., J. Pharm. Sci. 66, 1094 (1977).

6. Kwan, K. H., Higuchi, W. I., and Hofmann, A. F., J. Pharm. Sci. 67, 1711 (1978).

7. Patel, D. C., and Higuchi, W. I., J. Colloid Interface Sci. 74, 201 (1980).
8. Patel, D. C., and Higuchi, W. I., J. Colloid Interface Sci. 74, 211 (1980).

9. Patel, D. C., and Higuchi, W. I., J. Colloid Interface Sci. 74, 220 (1980).

10. Mazer, N. A., Benedek, G. B., and Carey, M. C., Gastroenterology 70, 998 (1976).

11. Mazer, N. A., Benedek, G. B., and Carey, M. C., Biochemistry 19, 601 (1980).

12. Higuchi, W. I., Su, C. C., Park, J. Y., Alkan, M. H., and Gulari, E., J. Phys. Chem. 85, 127 (1981).

13. Small, D. M., in "Molecular Association in Biological and Related Systems" (R. F. Gould, Ed.), Chem. Series 84, pp. 31-52. Washington, D. C., 1968.

14. Mukerjee, P., and Cardinal, J. R., J. Pharm. Sci. 65, 883 (1976).

15. Mazer, N. A., Carey, M. C., and Benedek, G. B., in "Micellization, Solubilization and Microemulsions" (K. L. Mittal, Ed.), Vol. 1, pp. 359-381; 383-402. Plenum, New York, 1977.

16. Chang, Y., and Cardinal, J. R., J. Pharm. Sci. 67, 174 (1978).

17. Becher, P., "Chemistry, Physics and Applications of Surface Active Substances, II. Physics and Physical Chemistry of Surface Active Substances," p. 621. Gordon \& Breach, New York, 1967.

18. Shinoda, K., "Principles of Solution and Solubility," pp. 157-199. Dekker, New York, 1978.

19. Kamel, A. M., Weiner, N. D., and Felmeister, A., J. Colloid Interface Sci. 35, 163 (1971).

20. Feld, K. M., Ph.D. thesis, The University of Michigan (1980).

21. Krevoy, M. M., and Wewerka, E. M., J. Phys. Chem. 71, 4150 (1967).

22. Keller, K. H., Canales, E. R., and Yum, S. I., J. Phys. Chem. 75, 379 (1971).

23. Gosting, L. J., and Morris, M. J., J. Amer. Chem. Soc. 71, 1998 (1949).

24. Henrion, P. N., Faraday Soc. Trans. 60, 72 (1964).

25. Kaganov, I. N., and Tuzhilkin, V. I., Chem. Abstr. 64, 19956 (1966).

26. Patel, D. C., Ph.D. thesis, The University of Michigan (1979).

27. Berthoud, A., J. Chim. Phys. 10, 624 (1912).

28. Kushner, L. M., and Hubbard, W. D., J. Phys. Chem. 58, 1163 (1954).

29. Mukerjee, P., J. Pharm. Sci. 60, 1528 (1971).

30. Robson, R. J., and Dennis, E. A., J. Phys. Chem. 81, 1075 (1977).

31. Elworthy, P. H., and Florence, A. T., Kolloid-Z. ZPolym. 204, 105 (1965).

32. Mukerjee, P., in "Solution Chemistry of Surfactants" (K. L. Mittal, Ed.), Vol. 1, p. 153. Plenum, New York, 1979.

33. Ogino, K., and Takeshita, N., Bull. Chem. Soc. Jpn. 53, 611 (1980).

34. Feld, K. M., and Higuchi, W. I., and Su, C. C., J. Pharm. Sci. 71, 182 (1982). 